\title{
Achieving Proportional Loss Differentiation Using Probabilistic Preemptive Burst Segmentation in Optical Burst Switching WDM Networks
}

\author{
Chee-Wei Tan ${ }^{2}$, Mohan Gurusamy ${ }^{1}$ and John Chi-Shing Lui ${ }^{2}$ \\ ${ }^{1}$ Electrical and Computer Engineering Dept., National University of Singapore, Singapore \\ ${ }^{2}$ Computer Science and Engineering Dept., The Chinese University of Hong Kong, Shatin, N.T., Hong Kong \\ Email: cwtan@ie.cuhk.edu.hk, elegm@nus.edu.sg, cslui@cse.cuhk.edu.hk
}

\begin{abstract}
We propose a Probabilistic Preemptive Burst Segmentation (PPBS) scheme to provide traffic classes with different Quality-of-service (QoS) requirements in Optical Burst Switching (OBS) networks. PPBS enables high priority bursts to preempt and segment low priority bursts in a probabilistic fashion. By tuning a preemptive parameter that can be instrumented locally on an OBS node, our scheme provides a flexible method to achieve service differentiation on an OBS switch for multiple prioritized traffic classes. We develop an analytical queueing model that captures the notion of increased bandwidth and parallel service using multiple wavelengths. It achieves $100 \%$ isolation between high and low priority classes and low loss probabilities in the single and multiple wavelength case. Specifically, our queueing model can achieve Proportional Differentiated Service (PDS) in terms of loss. Finally, we also compare the performance of PPBS with previous heuristic methods in achieving proportional loss differentiation using Long Range Dependent traffic models.
\end{abstract}

Index Terms- Optical Burst Switching, Burst segmentation, Quality-of-service (QoS), Proportional Differentiated Service

\section{INTRODUCTION}

Optical burst switching (OBS) [8], [9], [13] is a promising technique towards achieving optical packet switching in next generation IP over wavelength-division-multiplexing (WDM) networks. In this paper, we concentrate on the Just-EnoughTime (JET) ([8] pp. 357) reservation protocols where wavelength reservations are done in a one-way process, and the arrival and departure time of the data bursts are known in advance. Bursts are differentiated in the OBS network based on assigned priorities which are stored in the BHP. If the wavelength is successfully reserved, the arriving burst is switched optically through the OBS switches. However, if the requested wavelength is not available, the burst is said to be blocked or dropped.

Internet traffic can be largely categorized as high priority and low priority data because mission-critical and real-time applications like audio and video require a high Quality of Service (QoS). Supporting QoS (e.g., low delay and loss probability) at the WDM layer will facilitate a QoS-enhanced version of the next generation Internet [13]. Yoo et al. proposed a scheme which differentiates between classes of service based on the offset time assigned to each class [13]. The lowest priority class gets a base offset and every higher class has an extra offset time in addition to the base offset. Having a longer offset time would allow high priority class to reserve wavelengths in advance. For example, in order to achieve 95\% degree of isolation between two different traffic classes in wavelength reservation, the extra offset is proposed to be $3 \bar{L}$ where $\bar{L}$ is the mean burst length of the low priority class [13]. This scheme aims to achieve maximum isolation between different priority classes for service differentiation but this scheme results in longer delay and large buffer requirement at edge routers. Further, it may over-penalize low priority classes.

Alternative QoS models have been proposed that do not introduce extra offset time, hence no extra delay is incurred for high priority bursts. For example, the Probabilistic Preemption scheme (PPS) allows high priority bursts to preempt low priority burst in a probabilistic fashion but there is no isolation between priority classes [12]. The Prioritized Burst Segmentation (PBS) scheme is similar to conventional OBS except that conflicting parts are segmented to resolve wavelength contention [10]. The conflicting segment or the entire low priority burst may be dropped or routed to other links with free wavelengths using Deflection Routing (DR) which yields higher throughput than traditional JET-based OBS protocol [10].

Rather than providing absolute QoS guarantees, Proportional Differentiated Service (PDS) aims to achieve better performance for high priority class than low priority class with a fixed quality spacing, i.e., consistent service differentiation [2], [7]. Having a consistent performance spacing would allow the network opertator to legitimately charge higher priority class a higher tariff rate [7]. Furthermore, the PDS model specifies that consistent quality spacing should be met even in short timescales. Much work has been done in the context of proportional delay differentiation, for eg. in [2] and [7]. However, work on proportional differentiation in OBS networks have been few. Indeed, in [4], it has been envisioned by Dovrolis and Ramanathan that WDM technologies provide Internet Service Providers (ISP) the ability to lease the capacity of additional wavelengths from the backbone providers that owns the network fibers, when additional capacity is anticipated or encountered, in order to meet some form of PDS. However, designing and provisioning discretized capacity for a system wherein many classes share the same group of wavelengths 
to achieve some form of PDS remains an open question. In the context of OBS network, Chen et al. proposed an intentional dropping scheme to drop burst to maintain a predefined ratio spacing [5] but this scheme may result in low system utilization due to excessive dropping. Cankaya $e t$ al. proposed a partial preemptive technique to achieve PDS where only conflicting parts of the burst are dropped [1]. The results in [5] and [1] are promising, but are largely ad hoc in nature, and are based only on simulations.

The contributions of the paper are as follows: We develop an analytical model for probabilistic preemptive burst segmentation using Markov chains. Unlike a previous analytical approach in [10], our model captures the notion of increased bandwidth and parallel service using multiple wavelengths. A Probabilistic Preemptive Burst Segmentation (PPBS) scheme that preempts and segments low priority bursts in a probabilistic fashion is proposed. As compared to the extra offset timebased scheme, the degree of isolation in PPBS is $100 \%$. This scheme achieves complete isolation and flexibility for service differentiation, and offers benefits to network throughput. Most importantly, by appropriate tuning of control parameters, we show that PDS in terms of loss can be effectively achieved on a link with multiple wavelengths. Whereas previous analyses in related work [13], [10], [12] only consider two priority classes, our work focuses on the general multi-class analysis.

The rest of the paper is organized as follows. In Section II, we first develop a Markov model for PPBS in a link with a single wavelength, and subsequently, a stochastic model for a link with multiple wavelengths for two priority classes. In Section III, we analyze PDS using PPBS for two priority classes. In Section IV, we extend our results to an arbitrarily number of traffic classes, and we propose a measurementbased scheme to achieve PDS for a multi-class system. In Section V, we study the performance of PPBS using Long Range Dependence (LRD) traffic models, and we compare our results with previously proposed algorithms in achieving PDS. We conclude the paper in Section VI.

\section{A Probabilistic Preemptive Burst SEGMENTATION (PPBS) SCHEME}

In this section, we introduce the PPBS scheme. A burst can use any free wavelength regardless of its priority class. When a high priority burst arrives and no free wavelength is available, a control parameter associated with each traffic class allows an OBS node to use preemption (Fig. 1a) or segmentation (Fig. 1b) probabilistically. In segmentation, the part of the burst that remains is known as the truncated segment, and the conflicting part that is removed is known as the lost segment. Instead of dropping them, both the preempted burst in Fig. 1a and the lost segment in Fig. 1b may be assigned to other links with free wavelengths using Deflection Routing. There is no preemption within the same priority class, and only high priority bursts preempt low priority bursts. Allowing high priority bursts to get through at the expense of low priority bursts has the same

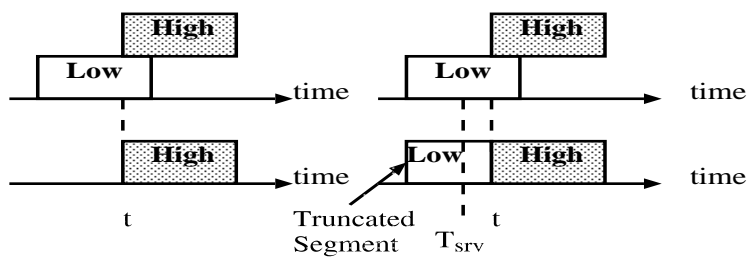

(a)

(b)

Fig. 1. Illustration of PPBS. (a) A low priority burst gets completely preempted. (b) A low priority burst gets segmented.

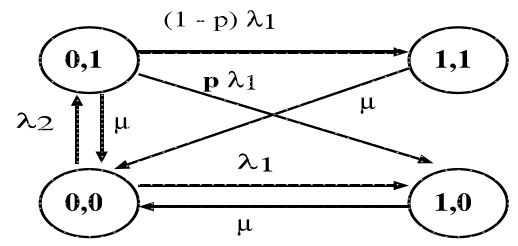

Fig. 2. A Markov chain analysis of PPBS

motivation as the extra offset time-based scheme ${ }^{1}$.

\section{A. Markovian Model with A Single Wavelength}

First, we consider two classes of traffic, i.e., 1 and 2, where class 1 (real-time traffic) has higher priority over class 2 (best-effort traffic) in resource reservation. Class 1 and Class 2 have arrival rates $\lambda_{1}$ and $\lambda_{2}$ respectively. Each class has a negative exponential service distribution of mean $\mu$. In addition, we assume as in [13] that all classes have equal basic offset time differences. The performance metric is the burst loss probability in a single OBS switch. We model the PPBS queueing system using a continuous time Markov chain whose state $\pi(i, j)$ is constituted by two discrete variables $(i, j)$ where $i$ and $j$ denote the number of high priority and low priority bursts respectively on a single wavelength. A low priority burst gets preempted completely or segmented with probability $p$ and $1-p$ respectively.

Fig. 2 shows the state transition diagram of the Markov chain. Solving for the limiting state probabilities in Fig. 2 and assuming $\mu=1$, we obtain the burst dropping probability of the high priority class, $P_{H}$, and low priority class, $P_{L}$ respectively, as

$$
P_{H}=\frac{\lambda_{1}}{1+\lambda_{1}} ; \quad P_{L}=\frac{\rho\left(1+\lambda_{1}\right)+p \lambda_{1}}{(1+\rho)\left(1+\lambda_{1}\right)}
$$

where $\rho$ is the total offered load and is defined as $\lambda / \mu$. Clearly, we have a $M / M / 1 / 1$ queueing system and we assume $\rho<$ 1 for stability. Note that we obtain PPS [12] by eliminating state $\pi(1,1)$ but PPBS makes sense because it also takes into account the possibility that burst preemption may occur after the low priority data burst is already in service, and such a case is likely to occur when burst lengths are relatively long on the average which is a feature of optical burst switching as indicated by $T_{s r v}$ in Fig. 1(b). Further, for a fixed $p, P_{L}$ is

\footnotetext{
${ }^{1}$ The motivations for complete isolation between traffi c classes are discussed in details in [13].
} 
always smaller than that in PPS [12]. If $p=0$, we have the PBS scheme ${ }^{2}$. Choosing an appropriate value for $p$ gives a flexible range of service differentiation, which is not possible in PBS. More importantly, the linear relationship in $p$ can provide Proportional Loss Differentiation as we shall show in the next section. The following theorem gives the mean of the segmented burst.

Theorem 1: For a single wavelength case with two priority classes, the mean of the truncated segment is $1 /\left(1+\lambda_{1}\right)$ and the mean of the lost segment is 1.0 .

Proof: Please refer to [14].

Remark: Since, $\lambda_{1}<1$, the mean of the truncated segment is always larger than $1 / 2$. Hence, a large percentage of low priority class can be salvaged in PPBS as compared to dropping the complete burst. Furthermore, a lower $P_{H}$ implies a higher mean of the truncated burst, i.e., a mean closer to 1.0.

\section{B. Stochastic Model with Multiple Wavelengths}

We now extend our results to the case of a link with multiple wavelengths. First, we need several results from [13]. It has been shown in [13] that the dropping probability of the classless OBS is given by the Erlang's loss formula $(M / G / k / k)$

$$
B(\rho, k)=\left(\rho^{k} / k !\right) /\left(\sum_{i=0}^{k} \rho^{i} / i !\right)
$$

where $k$ denotes the number of wavelengths. Also, by representing the average loss probability $P_{\text {all }}$ using a weighted sum of loss probability of each class, a conservation law $^{3}$ among two prioritized traffic classes is given in [13] by

$$
\lambda P_{\text {all }}=\lambda_{1} P_{H}+\lambda_{2} P_{L}^{\prime} .
$$

Now, $P_{\text {all }}$ is the average loss rate in a classless OBS system. Hence, $P_{\text {all }}=B(\rho, k)$. On the other hand, the two terms on the right hand side constitute a 2-class system with strict priority. Hence, we have $P_{H}=B\left(\lambda_{1}, k\right)$ and $P_{L}^{\prime}=$ $\left(\lambda B(\rho, k)-\lambda_{1} P_{H}\right) / \lambda_{2}$. The following results bounds the dropping probability of class 2 traffic $P_{L}$ in PPBS.

Lemma 1: For two classes of traffic in a link with general $k$ wavelengths in PPBS, $P_{L}$ is upper bounded by $P_{u p p}=P_{L}^{\prime}$ and lower bounded by $P_{l o w}=B(\rho, k)$.

Proof: Please refer to [14].

Using Lemma 1, we can obtain an approximation for the dropping probability of class 2 traffc, since $P_{\text {low }}$ and $P_{u p p}$ are only dependent on the traffic arrival rates $\lambda_{1}$ and $\lambda_{2}$, and are independent of $p$. Next, by observing that $P_{L}$ has a linear relationship with respect to $p$ between the bounds $P_{u p p}$ and $P_{\text {low }}$ for the single wavelength case, a formula for $P_{L}$ in a link with general $k$ wavelengths is given by the following theorem.

\footnotetext{
${ }^{2}$ For the PBS analysis, our result for the loss of the lower priority class, $P_{L}$ differs from that in [10] because the analysis in [10] assumes in addition that segmentation may occur within the same priority class by allowing the contending burst to always segment the burst that has been scheduled earlier. Hence, the loss of $P_{L}$ is higher in their analysis assuming a single node with $\mu=1$.

${ }^{3}$ In [13], the conservation law is conjectured to be true only when the traffi $\mathrm{c}$ load is high, but it is a valid relationship for all traffi $\mathrm{c}$ intensities in PPBS for $p=1$.
}

Theorem 2: For a 2-class system in a link with $k$ wavelengths, the dropping probability of class 1 traffic is determined by $B\left(\lambda_{1}, k\right)$, and the dropping probability of class 2 traffic is determined by

$$
P_{L}=p P_{u p p}+(1-p) P_{\text {low }} .
$$

Proof: Please refer to [14].

Observe that the closed form solutions of $P_{H}$ and $P_{L}$ are stated only in terms of the Erlang's loss formula. It is well known that the Erlang's loss model belongs to an insensitive queueing system: Eqn. (2) is valid regardless of the service time distribution. Hence, it is intuitive to expect that PPBS also belongs to this class of queueing system.

Corollary 1: PPBS exhibits the insensitivity property.

Proof: Please refer to [14].

Corollary 1 is important for an OBS network, because firstly, different burst assembly techniques may be employed at the edge which result in different burst service distribution seen by the scheduling node, and secondly, burst scheduling decision at the node can be made independent of the burst assembly technique being used.

\section{Proportional Loss DifFEREnTIATION FOR Two Classes}

Let $r_{1,2}$ be the required ratio of the average loss probability of class 2 traffic to that of class 1 traffic. Since the dropping probability of class 1 is independent of class 2 , we can provide proportional service differentiation for two prioritized traffic classes in $k$ wavelengths by adjusting the preemptive parameter $p$ with the following result.

Theorem 3: For a link with $k$ wavelengths, to achieve the specified ratio $r_{1,2}$, the preemptive parameter of PPBS can be set as $p=\left(r_{1,2} P_{H}-P_{\text {low }}\right) /\left(P_{\text {upp }}-P_{\text {low }}\right)$ where both $P_{\text {upp }}$ and $P_{l o w}$ are defined in the previous section. The achievable ratio $r_{1,2}$ is valid for $P_{\text {low }} / P_{H} \leq r_{1,2} \leq P_{u p p} / P_{H}$.

Proof: Theorem 3 is proved using Theorem 2 .

Notice that the feasible region, i.e., a region wherein the preemptive parameter $p$ is less than 1 , for a single wavelength using $p$ as the single degree of freedom is limited. In general, the width of the feasible region is proportional to

$$
\frac{\lambda_{1}}{\lambda_{2}}\left(\frac{B(\rho, k)}{B\left(\lambda_{1}, k\right)}-1\right) .
$$

Hence, increasing $k$ enlarges the feasible region. Also, the ratio of high priority to low priority class plays an important role in determining a wide feasible region. For $k=1$, the width is a constant that is only dependent on the total system utilization and, for general $k>1$, it is a function of load distribution. In Section IV-A, we shall show how the feasible region can be enlarged by taking into account the number of wavelengths allocated, and the number of competing classes.

\section{Analysis of Multiple Traffic Classes}

For the case of $k$ wavelengths, we extend the previous results for two prioritized traffic classes to general $M$ traffic classes. In particular, any high priority class can preempt and segment any low priority class with different probabilities. Let 
us define $p_{j i}, i=1, \ldots, M-1$ for all $j<i$ as the preemptive parameter that class $j$ preempts class $i$. Correspondingly, class $j$ segments class $i$ with probability $1-p_{j i}$. Let $\mathbf{p}_{i}$ be a column vector of size $M-1$ where the first $i-1$ components are the probabilities $p_{j i}$, while the remaining $M-i$ components are zero. Call $\mathbf{z}$ a column vector of size $M-1$ whose components are the input rate $\lambda_{j}$ for $1 \leq j \leq M-1$. By denoting the mean loss of class $i$ as $L_{i}\left(\mathbf{p}_{i}\right)$ or $L_{i}$ for brevity, we have

Theorem 4:

$$
L_{i}\left(\mathbf{p}_{i}\right)=R_{i}+\mathbf{p}_{i}^{T} \mathbf{z} S_{i}
$$

where

$$
R_{i}=B\left(\sum_{j=1}^{i} \lambda_{j}, k\right) ; \quad S_{i}=\left(R_{i}-R_{i-1}\right) / \lambda_{i} .
$$

Proof: Consider a particular class $i, 1<i \leq M$. We segregate class $i$ from all the classes with higher priorities than class $i$ by grouping them into a single group $G$. This has effectively reduced a multi-class analysis into one of a 2 -class system. We can therefore proceed to derive the loss probability of class $i$ due to preemption from the total traffic in group $G$ on class $i$ using results we have obtained in Section II-B. We have $p_{j i}, j<i, i=1, \ldots, M-1$ as the preemption parameters in $G$ on class $i$. Since we assume Poisson arrival inputs, the total sum of each high priority class that preempts class $i$, i.e., $\sum_{j=1}^{i-1} p_{j i} \lambda_{j}$, is also Poisson. It is straightforward to obtain the loss of class $i$ as

$$
\begin{aligned}
& B\left(\sum_{j=1}^{i} \lambda_{j}, k\right)+\left(\sum_{j=1}^{i-1} p_{j i} \lambda_{j} / \lambda_{i}\right)\left(B\left(\sum_{j=1}^{i} \lambda_{j}, k\right)-\right. \\
& \left.B\left(\sum_{j=1}^{i-1} \lambda_{j}, k\right)\right), i=1, \ldots, M .
\end{aligned}
$$

Hence, Theorem 4 follows from our definition of $\mathbf{p}_{i}$ and $\mathbf{z}$ with the symbol $\left({ }^{T}\right)$ denoting the transpose operator.

Similar to the 2-class system analysis, Theorem 4 shows that the loss of class $i$ is linear in $\mathbf{p}_{i}$. The number of classes and the corresponding offered load are therefore key design parameters used for instrumenting the loss probabilities. Since, in a dynamically varying load situation, we cannot control the offered load, different loss probabilities can be specified by a proper choice of the parameter vector $\mathbf{p}_{i}$ for each class $i$. Below, we shall drop the subsript $i$ for brevity since it is understood that $\mathbf{p}_{i}$ is used uniquely with class $i$ only.

Special case: When we have strictly preemption, all the preemption parameters are 1 for all classes. In general, the conservation law introduced in section II-B can be extended to relate the weighted loss of each class $i$ for multiple traffic classes. Therefore, we have the following result.

Theorem 5: The conservation law holds for multiple traffic classes $M$ in a link with $k$ wavelengths using a preemption vector $\mathbf{p}=\mathbf{I}$ where $\mathbf{I}$ denotes the column vector with the corresponding components unity, i.e.,

$$
\rho B(\rho, k)=\sum_{j=1}^{M} \lambda_{j} L_{j}(\mathbf{I})
$$

Proof: The theorem is proved using Theorem 4.

Remark: It is interesting to note that, in the above system configuration for $\mathbf{p}=\mathbf{I}$, there exists $M-1$ sets of conservation law relationship. An example that illustrates this point is as follows. Consider a 3-class system with $k$ wavelengths, then both Class 1 and Class 2 will obey a set of conservation law such that $\left(\rho_{1}+\rho_{2}\right) R_{2}=\sum_{j=1}^{2} \lambda_{j} L_{j}(\mathbf{I})$, and all the three classes will obey another set of conservation law such that $\rho R_{3}=\sum_{j=1}^{3} \lambda_{j} L_{j}(\mathbf{I})$ where $R_{i}$ is given in Theorem 4. Since $R_{M}=B(\rho, k)$, Theorem 5 is the only relationship that relates the total system utilization and the loss of each class together.

\section{A. Proportional loss differentiation in multiple traffic classes}

According to the multi-class Proportional Loss Differentiation model [3], the average class loss rates are fixed to:

$$
\frac{L_{i}}{L_{j}}=\frac{\sigma_{i}}{\sigma_{j}}, \quad 1 \leq i, j \leq M,
$$

where the parameters $\sigma_{i}$ are the Loss rate Differentiation Parameters (LDPs), and they are ordered as $\sigma_{1}<\cdots<\sigma_{M}$. To achieve Eqn. 7, we define a fixed proportional loss ratio between each low priority class $i, 1<i \leq M$ and the highest priority class, $r_{1, i}$, i.e., $r_{1, i}=\sigma_{i} / \sigma_{1}$. Now, similar to section III, the proportional loss ratios $r_{1, i}$ between each class can be achieved by selecting a proper $\mathbf{p}$. The difference to section III lies in the fact that, beside using different number of wavelengths $k$, we have an additional $i-1$ degrees of freedom for each class $i$ to enlarge the feasible region. Furthermore, increasing $M$ enlarges the feasible region. Our proposed scheme for achieving PDS is as follows: From Theorem 4 and eqn. (4), we see that feasible $\mathbf{p}$ vectors are functions of traffic loading. Hence, each PPBS node measures the offered load for each class periodically to obtain $\mathbf{z}$ (or obtain $\mathbf{z}$ from the BHP if the rate information is encoded during the burst assembly process). For this purpose, we suggest the jumping window scheme ${ }^{4}$ as in [7]. Using $\mathbf{z}$ and selecting $k$ wavelengths from a pool of wavelengths, we dynamically adapt a parameter square matrix $\mathbf{P}$ where $\mathbf{P}=\left[\mathbf{p}_{2}, \ldots, \mathbf{p}_{M}\right]$ to maintain $r_{1, i}, i=2, \ldots, M$. Since each component in $\mathbf{P}$ cannot be more than 1 , the feasible ratio range for a fixed $k$ is

$$
R_{i} / P_{H} \leq r_{1, i} \leq\left(S_{i} \sum_{j=1}^{i-1} \lambda_{j}+R_{i}\right) / P_{H}, i=2, \ldots, M
$$

where $P_{H}$ is the loss of the highest priority class. If the ratio is beyond this region, we adjust $k$ until the target ratio $r_{1, i}$ is met. However, the feasible regions for different $k$ are disjointed, hence, for a fixed $M$, some $r_{1, i}$ cannot be achieved using PPBS for a given load distribution.

\section{Performance Study}

In this section, we consider LRD traffic models for our simulations. There is evidence that LRD will remain a salient property of network traffic even as network characteristics such as bandwidth and topology evolve over time, hence

\footnotetext{
${ }^{4}$ Please refer to [7] for a detailed discussion of the jumping window scheme
} 


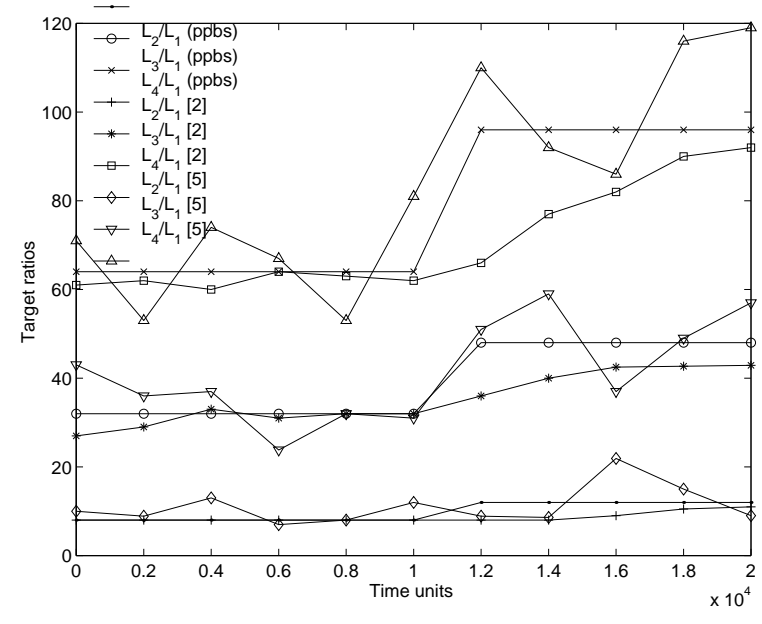

Fig. 3. Loss ratios achieved with a change in desired loss ratios at time $=$ 10000 units.

it is necessary to evaluate the impact of LRD traffic on burst loss probabilities in PPBS [6]. LRD traffic is modeled by a superposition of 64 On/Off sources with On and Off periods distributed according to a Pareto distribution with shape parameter 1.3. The simulation results are obtained for a single OBS switch with an output link having multiple wavelengths. The mean service time is taken to be the unit of time and the service times of packets in each class follow the same exponential distrbution with unit mean unless otherwise stated. Burst dropping probability is the performance metric.

\section{A. Experiment (Comparison between PPBS and other schemes)}

We compare the performance of PPBS with the partial preemptive scheme in [1] and the intentional dropping scheme in [5]. For all the schemes, we use the jumping window scheme [7] to periodically monitor the traffic load. For both schemes in [1] and [5], low priority class burst is selected to be partially preempted or intentionally dropped respectively at the end of each window $W$, while the PPBS scheme dynamically adapts $\mathbf{P}$. We use 3 wavelengths and 4 classes. The total system utilization $\rho$ is set at 0.4 with even Poisson load distribution. The desired target ratios are first selected as $r_{1,2}=8, r_{1,3}=$ $32, r_{1,4}=64$, and changed to $r_{1,2}=12, r_{1,3}=48$ and $r_{1,4}=$ 96 at $1 \times 10^{4}$ time units with $W$ selected as 2000 time units (a medium window size). Fig 3 shows the short-term loss ratio with time. The achievable loss ratios $L_{i} / L_{1}, i=2,3,4$ for the PPBS scheme, partial preemptive scheme in [1] and intentional dropping scheme in [5] are indicated in Fig. 3, respectively. Dynamic adjustment of $\mathbf{P}$ using the jumping window scheme is effective, while the methods in [1] and [5] cannot achieve the target ratios most of the time. The method in [5] results in large dropping of low priority bursts and low bandwidth utilization, whereas the method in [1] cannot maintain the desired ratios effectively when there is a change at time $=10000$ units. In comparison to [1] and [5], we conclude that our scheme can more efficiently and robustly achieve multi-class proportional loss differentiation under a variety of operating conditions, i.e., different system utilizations, different number of traffic classes, and different number of load distributions. More details on other experimental results on PPBS can be found in [14].

\section{CONCLUSIONS}

We developed an analytical model to evaluate the performance of optical burst switching for prioritized traffic classes using a Probabilistic Preemptive Burst Segmentation (PPBS) queueing model. We showed that our results are applicable for a link with an arbitrarily number of wavelengths and number of traffic classes with general service distribution. We also showed that PPBS is more flexible than PBS by providing diverse loss differentiation, and performs significantly better than PPS in terms of loss rates. Most importantly, we presented the feasibility of achieving proportional loss differentiation using PPBS which is more superior than previous schemes. Finally, experiments showed that PPBS is promising using aggregated bursty traffic models.

\section{REFERENCES}

[1] H. C. Cankaya, S. Charcranoon and T. S. El-Bawab, "A preemptive scheduling technique for OBS networks with service differentiation", Proc. IEEE Globecom 2003, November 2003.

[2] C. Dovrolis, D. Stiliadis and P. Ramanathan, "Proportional differentiated services: Delay differentiation and packet scheduling", IEEE/ACM Transactions on Networking, vol. 10, No. 1, February 2002.

[3] C. Dovrolis and P. Ramanathan, "Proportional differentiated services, Part II: Loss rate differentiation and packet dropping", Proc. IEEE IWQoS, Pittsburgh PA, June 2000.

[4] C. Dovrolis and P. Ramanathan, "Class provisioning using proportional delay differentiation", Proc. Scalability and Traffic Control in IP Networks Conference (SPIE ITCOM302), August 2001.

[5] Y. Chen, M. Hamdi, D. H. K. Tsang and C. Qiao, "Proportional QoS provision: A uniform and practical solution”, IEEE ICC 2002, vol. 4, pp. 2363 -2367, May 2002.

[6] A. Ge, F. Callegati and L. S. Tamil, "On optical burst switching and self-similar traffi c" IEEE Communications Letter, vol. 4, No. 3 , March 2000.

[7] M. K. H. Leung, J. C. S. Lui and D. K. Y. Yau, "Adaptive proportional delay differentiated services: Characterization and performance evaluation", IEEE/ACM Transactions on Networking, vol. 9, No. 6, December 2001.

[8] C. S. R. Murthy and G. Mohan, "WDM Optical Networks: Concepts, Design and Algorithms", Prentice Hall, November 2001.

[9] J. S. Turner, "Terabit burst switching", Journal of High Speed Networks, vol. 8, No. 1, pp. 3-16, 1999.

[10] V. M. Vokkarane and J. P. Jue, "Prioritized burst segmentation and composite burst assembly techniques for QoS support in optical burst-switched networks", IEEE Journal on Selected Areas in Communications, vol. 21, no. 7, pp. 1198-1209, September 2003.

[11] Y. Xiong, M. Vandenhoute and H. C. Cankaya, "Control architecture in optical burst-switched WDM networks", IEEE Journal on Selected Areas in Communications, vol. 18, No. 10, October 2000.

[12] L. Yang, Y. Jiang and S. Jiang, "A probabilistic preemptive scheme for providing service differentiation in OBS networks", Proc. IEEE Globecom 2003, November 2003.

[13] M. Yoo, C. Qiao and S. Dixit, "QoS performance in IP over WDM networks", IEEE Journal on Selected Areas in Communications, vol. 18, No. 10, pp. 2062-2071, October 2000.

[14] C.W. Tan, G. Mohan and J.C.S. Lui, "Achieving multi-class service differentiation in WDM optical burst switching networks: A probabilistic preemptive burst segmentation scheme", NUS Technical Report, July 2004. 